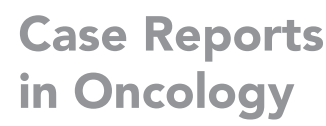

\title{
Benign-Appearing Glucagonoma Undergoing Malignant Transformation after a Prolonged Period
}

\author{
Mohamed K.M. Shakir ${ }^{a} \quad$ Ismail C. Ebrahim ${ }^{a}$ Andrea N. Snitchler ${ }^{b}$ \\ Vinh Q. Mai ${ }^{a}$ Thanh D. Hoang ${ }^{\text {a }}$ \\ aDepartment of Endocrinology, Walter Reed National Military Medical Center, Bethesda, \\ MD, USA; b Department of Pathology, Walter Reed National Military Medical Center, \\ Bethesda, MD, USA
}

\author{
Keywords \\ Glucagonoma $\cdot$ Malignant transformation
}

\begin{abstract}
Glucagonoma are rare neuroendocrine tumors arising in the Langerhans islets of the pancreas. We report a patient with glucagonoma differentiation into a malignant form after 13 years. A 63-year-old asymptomatic man was evaluated for an enhancing lesion at the distal pancreas. Physical examination was normal. Laboratory values were normal except for an elevated serum glucagon level (206 pg/mL, Ref. 50-150). Somatostatin and other tumor markers were normal. A PET scan confirmed abnormal uptake at the distal pancreas, correlating with a CT scan. The patient underwent laparoscopic distal pancreatectomy with resection of a 2.6$\mathrm{cm}$ mass which predominantly expressed glucagon. Serum glucagon levels normalized immediately postoperatively. He remained asymptomatic for 13 years with normal blood glucose, glucagon, and chromogranin levels and normal surveillance MRI scans. Thirteen years following surgery, an elevated serum glucagon level $(230 \mathrm{pg} / \mathrm{mL})$ was observed. At this time he also remained asymptomatic. Abdominal MRI and a PET scan revealed hepatic lesions. Biopsy of the hepatic lesion confirmed metastatic glucagonoma. The patient was treated with lanreotide, which normalized the serum glucagon levels, and the tumor size remained stable for 12 months of follow-up. Complete remission without any treatment for more than 13 years confirmed the benign course of the glucagon-secreting tumor. The precipitating factors are unknown. This case highlights the importance of continuous monitoring of neuroendocrine tumors even beyond 10 years.




\section{Introduction}

Glucagonoma is a slow-growing alpha-cell tumor of the pancreatic islet of Langerhans. It may appear as a benign, localized tumor, but at least $50 \%$ of patients will have metastatic disease when diagnosed [1-4]. Rarely, glucagon-secreting tumors may occur in association with other tumors in multiple endocrine neoplasia syndrome, although these tumors compose less than $3 \%$ of glucagonoma cases [5]. These tumors typically occur in the distal pancreas and may vary in size from a subcentimeter size to $2.5 \mathrm{~cm}$ or larger [1-5].

Generally, patients with glucagonoma may present with symptoms of weight loss and new-onset diabetes mellitus; however, these tumors may also cause glucagonoma syndrome, mainly characterized by necrolytic migratory erythema, weight loss, and diabetes mellitus. We report a rare case of a 2.6-cm glucagon-producing tumor in a 77-year-old male. Following pancreatic tumor resection, the patient remained asymptomatic, but 13 years later the patient developed hepatic metastasis, which was detected by periodic radiology screening and elevated serum glucagon levels.

\section{Methods}

All laboratory and imaging studies were performed at Walter Reed National Military Medical Center, Bethesda, MD, USA.

\section{Case Report}

A 63-year-old man had initially been evaluated 13 years ago for an enhancing mass of 2.6 $\times 1.9 \times 2.2 \mathrm{~cm}$ at the pancreatic tail (Fig. 1a), which was noted on abdominal CT during workup of self-limiting abdominal discomfort. The patient also reported a history of hyperlipidemia, hypertension, and gastroesophageal reflux. He had no family history of endocrine disorders. His medications included atorvastatin, atenolol, and ezetimibe. He had no history of weight loss, poor appetite, bloating, nausea, vomiting, or skin rashes. Physical examination revealed normal vital signs and normal heart, lung, and abdominal examination results. Skin examination showed no dermatologic lesions. The initial laboratory values are shown in Table 1.
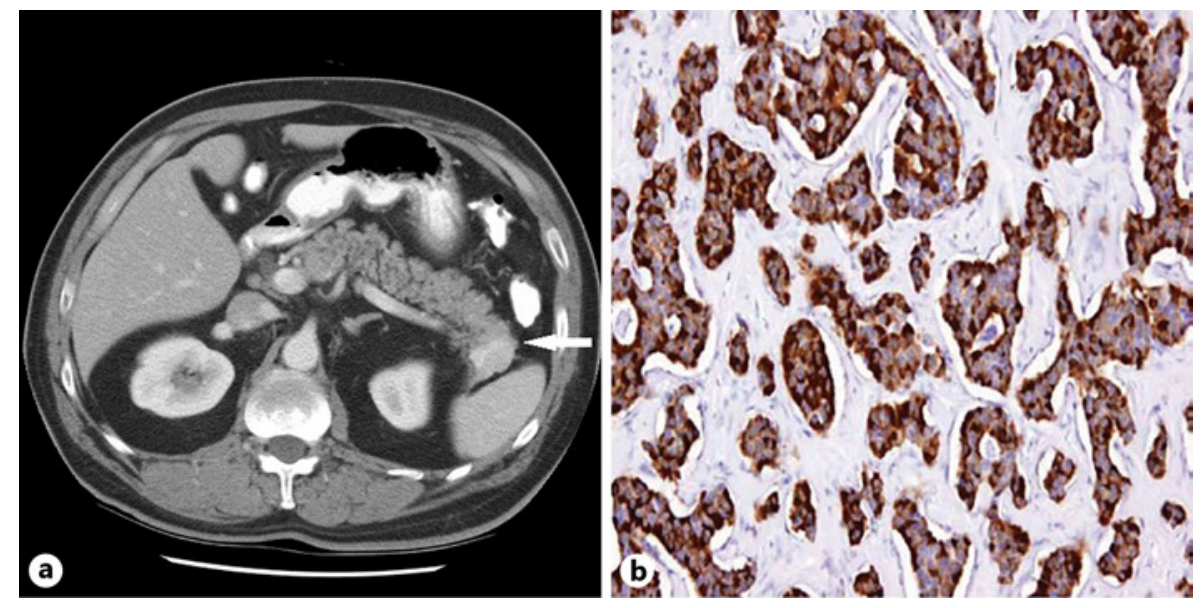

Fig. 1. a Abdominal CT showing an enhancing mass of $2.6 \times 1.9 \times 2.2 \mathrm{~cm}$ at the pancreatic tail. b Immunohistochemical staining was positive for glucagon. 
Shakir et al.: Glucagonoma Undergoing Malignant Transformation

Table 1. Laboratory results of various laboratory tests, before and after pancreatic glucagonoma resection, performed when the patient presented with hepatic metastasis, and laboratory tests performed 2 months following treatment with lanreotide

\begin{tabular}{lcccrr}
\hline Tests (fasting) & Preoperative & Postoperative & $\begin{array}{l}\text { At the diagnosis of } \\
\text { hepatic metastasis }\end{array}$ & $\begin{array}{c}\text { Lanreotide } \\
\text { therapy }\end{array}$ & $\begin{array}{c}\text { Reference } \\
\text { range }\end{array}$ \\
\hline Glucagon, pg/mL & 206 & 50 & 225 & 89 & $50-150$ \\
Glucose, $\mathrm{mg} / \mathrm{dL}$ & 98 & 91 & 108 & 109 & $74-106$ \\
Insulin, $\mathrm{mIU} / \mathrm{mL}$ & 10.5 & 6.2 & 17.8 & 7.2 & $2.6-24.9$ \\
Gastrin, $\mathrm{pg} / \mathrm{mL}$ & 129 & 42 & 37 & $0-115$ \\
Pancreatic polypeptide, pg/mL & 129 & 294 & 214 & 168 & $0.0-418.0$ \\
Vasoactive intestinal polypeptide, ng/mL & 33 & 24.4 & 29.7 & 32.4 & $0-58.8$ \\
Chromogranin, ng/mL & 14 & 8.5 & 10 & 95.3 & $0-101.8$ \\
\end{tabular}

Fig. 2. Graph showing serum glucagon levels over time.

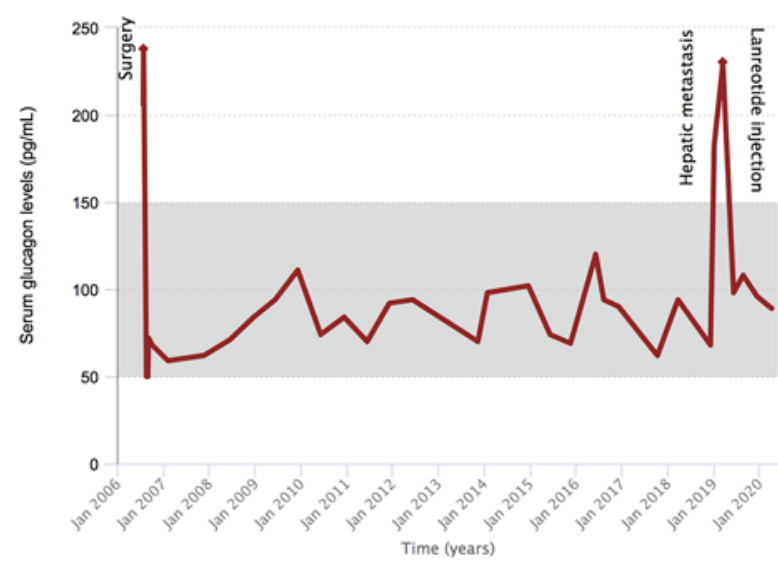

Note, shaded area represents normal levels (50-150)

Glucagonoma was suspected on the basis of elevated glucagon levels $(206 \mathrm{pg} / \mathrm{mL}$; Ref. 50-150; Table 1). Somatostatin and other tumor markers, including CA 19-9, carcinoembryonic antigen, and alpha-fetoprotein, were normal. Positron emission tomography (PET) and octreotide scintigraphy confirmed an abnormal uptake at the distal pancreatic tail (SUV of 1.2, compared to the rest of the pancreas with an SUV of 0.7), correlating with the CT scan findings. The patient underwent laparoscopic distal pancreatectomy with resection of a 2.6- $\mathrm{cm}$ mass that predominantly expressed glucagon and a lesser amount of gastrin with immunohistochemical staining (Fig. 1b). There was also small-vessel invasion and evidence of tumor extension into the peripancreatic fat and with neoplastic cells reacting strongly to chromogranin and glucagon. After surgical resection, the serum glucagon level in the peripheral venous blood and intraoperative splenic vein dropped to $<50 \mathrm{pg} / \mathrm{mL}$, confirming successful removal of the tumor. Additional laboratory tests were performed, which excluded multiple endocrine neoplasia 1 syndrome.

The patient remained asymptomatic for the next 13 years. During this time period, he underwent periodic screening for recurrence of glucagonoma by measuring serum glucagon levels (Fig. 2) and periodic abdominal MRI, and these tests were completely negative. After 13 years of follow-up, the serum glucagon level rose modestly to $225 \mathrm{pg} / \mathrm{mL}$ (Fig. 2), and abdominal MRI done at this time revealed a hepatic lesion, demonstrating arterial-phase hyperenhancement, contrast washout, and an enhancing pseudocapsule, suspicious for 

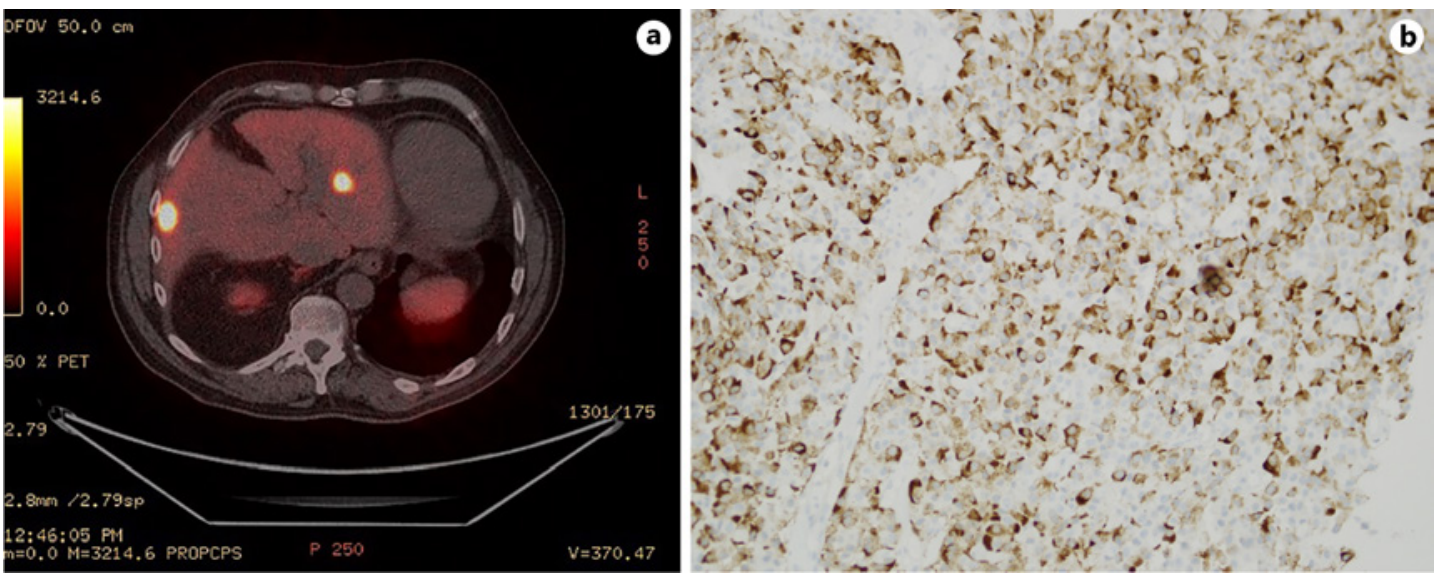

Fig. 3. a PET scan showing intense uptake in multiple hepatic lesions. b Immunostaining of the tissue specimen confirmed glucagonoma.

hepatic metastatic disease (Fig. 3a). The PET scan showed intense gallium-68 DOTATATE uptake in multiple hepatic lesions with more mild scattered osseous activity in the ribs and right proximal femur, concerning somatostatin receptor-expressing metastatic disease (Fig. 3a). The liver biopsy showed a metastatic well-differentiated neuroendocrine tumor. On immunohistochemistry, the neoplastic cells exhibited immunopositivity for chromogranin and synaptophysin and immunonegativity for Hep Par 1, CK7, and CK20, further supporting the diagnosis. Additionally, immunostaining of the tissue specimen confirmed glucagonoma (Fig. 3b), and the tumor in the current specimen was morphologically consistent with metastatic disease derived from the previously reported well-differentiated glucagonoma of the pancreas (Fig. 1b). A glucose tolerance test done at this time confirmed impaired glucose tolerance, and hemoglobin $A_{1 c}$ was 6.3\% (Ref. 4.0-6.0). A serum amino acid panel resulted normal.

The patient was treated with lanreotide $120 \mathrm{mg}$ subcutaneously every 4 weeks, resulting in normalized glucagon levels (Fig. 2). The treatment was continued for the next 12 months, and the hepatic lesions stabilized and the serum glucagon levels remained normal while the patient continued remaining asymptomatic. He was also receiving denosumab treatment because of the skeletal metastasis. Presently, the plan is to initiate peptide receptor radioligand therapy with Lutathera (lutetium ${ }^{177} \mathrm{Lu}$ DOTATATE) if he shows a poor response to lanreotide therapy, as shown by serum glucagon levels and/or progression of hepatic metastasis.

\section{Discussion}

Glucagon-secreting tumors, which account for $2 \%$ of islet cell tumors, are rare neuroendocrine pancreatic tumors with an estimated incidence of 1 in 20 million [1-7]. Although the diagnostic criteria for glucagonoma have already been established by Stacpoole [2], its rare incidence often hampers diagnosis and surgical intervention, especially when atypical manifestations are present. It is also important to realize the fact that the median time between the onset of glucagonoma and diagnosis varies from 3 to 4 years [6]. Of the various clinical features, necrolytic migratory erythema most likely can lead to an early diagnosis [6]. In our patient, the diagnosis was established by an incidental finding of a pancreatic mass occurring 
in association with modestly elevated serum glucagon levels. Furthermore, additional manifestations of glucagonoma may include glossitis, diarrhea, thromboembolic complications, and psychiatric manifestations [8,9]. Less common manifestations include heart failure, cardiomyopathy, and paraneoplastic features associated with tumoral secretion of proglucagon-derived GLP-1 and GLP-2 peptides, which may result in hyperinsulinemic hypoglycemia and marked gastrointestinal dysfunction [9]. Generally, the diagnosis of glucagonoma is considered with significant hyperglucagonemia $(>500 \mathrm{pg} / \mathrm{mL})$. However, our patient had no clinical features of glucagonoma, and this may have been related to lower levels of serum glucagon. Moderately elevated serum glucagon levels can be seen in hepatic cirrhosis, prolonged fasting, renal failure, and familial hyperglucagonemia; these disorders were excluded in our patient.

In a recent report, Eldor et al. [10] reported on 23 patients with glucagon-secreting tumors seen over 20 years. In this series of cases, only $50 \%$ of the patients had symptoms, and, intriguingly, no correlation between serum glucagon levels and the hormone-related syndrome was apparent [10]. This discrepancy may have resulted from overdiagnosis owing to the identification of immunoreactive glucagon fractions with reduced activity [10-12].

Glucagon-secreting tumors are usually diagnosed based on symptoms and elevated serum glucagon levels. Generally, glucagon levels $>500 \mathrm{pg} / \mathrm{mL}$ are seen in patients with glucagon-secreting tumors, and cross-sectional imaging of the pancreas (CT or MRI) can be performed. Since the majority of pancreatic neuroendocrine tumors are characterized by the presence of somatostatin receptors along the cell membrane, functional imaging using the somatostatin analog can detect the tumor. Although these scans can provide functional information, they have several limitations [13]. A somatostatin analog using ${ }^{98}$ Ga-DOTATATE PET/CT has higher sensitivity, specifically compared to octreotide scanning. Since neuroendocrine tumors of the pancreas are associated with alanine precursor uptake decarboxylase, ${ }^{18} \mathrm{~F}$-DOPA PET offers higher sensitivity than either CT or octreotide scanning alone [13]. ${ }^{18} \mathrm{~F}$-DOPA PET scanning also provides more relevant information for the clinical management of patients. Our patient had a PET scan when he developed hepatic metastasis, and this scan corresponded to MRI findings and thus guided the somatostatin analog therapy.

Surgery, involving complete resection of the tumor, is currently the only method to cure glucagonoma. In patients with hepatic metastasis, transarterial embolization and radiofrequency ablation can be utilized. Somatostatin analogs are useful in these patients. Chemotherapy such as with temozolomide, capecitabine, sunitinib, and everolimus can be used in patients with metastatic disease $[14,15]$. In a meta-analysis, Satapathy and Mittal [15] concluded that treatment with ${ }^{177}$ Lu-DOTATATE led to a better objective response, disease control rate, and longer progression-free survival than treatment with everolimus. Furthermore, ${ }^{177} \mathrm{Lu}$-DOTATATE therapy had a better safety profile [15]. However, in our patient, lanreotide treatment was initiated when hepatic metastases were detected, and the patient presently remains asymptomatic with no progression of hepatic metastases.

Our patient had microscopic tumor extension initially. However, the most interesting part of our case is the recurrence of the tumor after a prolonged period of time while the patient was remaining asymptomatic and having normal serum glucagon levels. Current clinical guidelines recommend that following tumor resection these patients be followed for a maximum duration of 10 years. However, based on our case, a longer period of surveillance may be needed. The exact pathogenesis of malignant transformation after a prolonged period of time remains unknown.

\section{Karger's}




\section{Case Reports in Oncology}

Case Rep Oncol 2020;13:1109-1115

\begin{tabular}{l|l}
\hline DOI: $10.1159 / 000509641$ & $\odot 2020$ The Author(s). Published by S. Karger AG, Basel
\end{tabular} www.karger.com/cro

Shakir et al.: Glucagonoma Undergoing Malignant Transformation

\section{Acknowledgements}

Ms. Shamla K. Shakir is acknowledged for editorial assistance, and Mr. Jay Mcdaniel for the graphic illustrations.

\section{Statement of Ethics}

This manuscript has been cleared by the institutional review board. The patient has given written informed consent to publish the case (including publication of images).

\section{Conflict of Interest Statement}

The authors have no multiplicity of interest to disclose. The views expressed in this article are those of the authors and do not reflect the official policy of the Department of Army/Navy/Air Force, Department of Defense, or US Government.

\section{Funding Sources}

No funding was received.

\section{Author Contributions}

All individuals who qualify as authors have been listed; each has participated in the conception and design of this work, the analysis of data, the writing of the document, and the approval of the submission of this version. I.C. Ebrahim wrote the draft. T.D. Hoang, V.Q. Mai, and M.K.M. Shakir reviewed and critically edited the paper. A.N. Snitcher reviewed the paper and provided the histology slides.

\section{References}

1 Leichter SB. Clinical and metabolic aspects of glucagonoma. Medicine (Baltimore). 1980;59(2):100-13.

2 Stacpoole PW. The glucagonoma syndrome: clinical features, diagnosis, and treatment. Endocr Rev. 1981; 2(3):347-61.

3 Wermers RA, Fatourechi V, Wynne AG, Kvols LK, Lloyd RV. The glucagonoma syndrome. Clinical and pathologic features in 21 patients. Medicine (Baltimore). 1996;75(2):53-63.

4 Van Beek AP, de Haas ER, van Vloten WA, Lips CJ, Roijers JF, Canninga-van Dijk MR. The glucagonoma syndrome and necrolytic migratory erythema: a clinical review. Eur J Endocrinol. 2004;151(5):531-7.

5 Henopp T, Anlauf M, Schmitt A, Schlenger R, Zalatnai A, Couvelard A, et al. Glucagon cell adenomatosis: a newly recognized disease of the endocrine pancreas. J Clin Endocrinol Metab. 2009;94(1):213-7.

6 Wei J, Lin S, Wang C, Wu J, Qian Z, Dai C, et al. Glucagonoma syndrome: a case report. Oncol Lett. 2015;10(2): 1113-6.

7 Burneikis T, Krishnamurthy VD. Glucagonoma. In: Docimo S Jr, Pauli E, editors. Clinical algorithms in general surgery. Cham: Springer; 2019. p. 457-9.

8 Mai VQ, Jones RC, Dickert JM, Clyde PW, Shakir KM. Plasma glucagon levels suppressed by a glucose load in a man with incidental pancreatic glucagonoma. Endocr Pract. 2007;13(7):780-4.

9 Albrechtsen NJW, Challis BG, Damjanov I, Holst JJ. Do glucagonomas always produce glucagon? Bosn J Basic Med Sci. 2016;16(1):1-7.

10 Eldor R, Glaser B, Fraenkel M, Doviner V, Salmon A, Gross DJ. Glucagonoma and the glucagonoma syndrome cumulative experience with an elusive endocrine tumour. Clin Endocrinol (Oxf). 2011;74(5):593-8. 
11 Kindmark H, Sundin A, Granberg D, Dunder K, Skogseid B, Janson ET, et al. Endocrine pancreatic tumors with glucagon hypersecretion: a retrospective study of 23 cases during 20 years. Med Oncol. 2007;24(3):330-7.

12 Recant L, Perrino PV, Bhathena SJ, Danforth DN, Lavine RL. Plasma immunoreactive glucagon fractions in four cases of glucagonoma: increased “large glucagon-immunoreactivity." Diabetologia. 1976;12(4):319-26.

13 Fathala A, Al Qahtani MH, Abouzied MM. Molecular imaging of a glucagonoma with 18F-FDG PET/CT and 68Ga-DOTATATE PET/CT imaging: a case report and review of the literature. Radiol Case Rep. 2020;15(1): 19-22.

14 de Mestier L, Walter T, Evrard C, de Boissieu P, Hentic O, Cros J, et al. Temozolomide alone or combined with capecitabine for the treatment of advanced pancreatic neuroendocrine tumor. Neuroendocrinology. 2020; 110(1-2):83-91.

15 Satapathy S, Mittal BR. 177Lu-DOTATATE peptide receptor radionuclide therapy versus everolimus in advanced pancreatic neuroendocrine tumors: a systematic review and meta-analysis. Nucl Med Commun. 2019;40(12):1195-203. 